\title{
A New Approach to Rough Vector Spaces
}

\author{
Shahad T.Almohammadi ${ }^{1}$, Cenap Özel ${ }^{* 2}$ \\ ${ }^{1}$ Department of Mathematics, (KAU) King Abdulaziz University, P.O. Box 80203, Jeddah 21589, Saudi Arabia \\ and Department of Mathematics, University of Jeddah, P.O. Box 80327, Jeddah 21589, Saudi Arabia \\ 2 Department of Mathematics, (KAU) King Abdulaziz University, P.O. Box 80203, Jeddah 21589, Saudi Arabia \\ ${ }^{1}$ shtlmd.93@gmail.com, stalmohammadi@uj.edu.sa, ${ }^{2}$ cenap.ozel@gmail.com, cozel@kau.edu.sa
}

\begin{abstract}
In this article, we will introduce the concept of rough vector spaces using upper approximation, and some algebraic results will be proven. Additionally, this concept will be combined with the concept of topological space.
\end{abstract}

Keywords: Rough set, lower approximation, upper approximation, approximation space, vector Spaces, topological space, topological vector space.

2010 MSC No: 15A03, 03E99

\section{Introduction}

In 1982, Z. Pawlak has proposed approximate operations on a set which were the beginning of rough set theory.[5] Actually the rough set theory is not new, it is just an expansion of the set theory to deal with incomplete information systems. It is based on the approximation space which is described by an ordered pair of nonempty set and equivalence relation $(U, R)$ called the universe and indiscernibility relation, respectively. Here the equivalence classes of a universe are called elementary sets and their finite union is called a composed set. Any subset of the universe in rough theory is represented by two sets, lower and upper approximations. The lower approximation of a given subset is the largest composed set which contained in the given set, and on the other hand, the upper approximation is the smallest composed set which contains the given set. Previous researches have established the most importance algebraic structures rough groups [2], rough rings [3], and rough modules [4].

In this article, we will generalize the notion of rough groups in [2] to construct the notion of rough vector spaces. Furthermore, some algebraic structures of rough vector spaces will be proven.

The organization of the paper is the following way: In Section 2 the elementary of rough set theory and some informations about rough group theory defined by Biwas and Nanda [2] will be given. In Section 3 we define and investigate rough vector spaces and some interesting examples will be given. In last part, we will give some further theory of rough vector spaces such as quotients, cosets, direct sums. Furthermore, in Section 4 we combine the concept that we define in the third section of rough vector space with the concept of topological space, called topological rough vector spaces.

\footnotetext{
${ }^{*}$ Corresponding author. CenapÖzel

cenap.ozel@gmail.com
} 
This paper is produced from the master thesis of the first author under King Abdulaziz University.

\section{Preliminaries}

The basic notions of the theory of rough set and rough groups will be shown, for more details $[2,5]$.

Definition 1. A pair $(U, R)$ of a nonempty set $U$ and an equivalence relation $R$ is said to be an approximation space.

Definition 2. Let $X$ be any subset of an approximation space $(U, R)$. Then $X$ described by the pair $(\underline{A p p}(X), \overline{A p p}(X))$ of lower and upper approximations respectively, such that

$$
\begin{aligned}
& \underline{A p p}(X)=\bigcup_{x \in U}\left\{[x]_{R}:[x]_{R} \subseteq X\right\}, \text { and } \\
& \overline{A p p}(X)=\bigcup_{x \in U}\left\{[x]_{R}:[x]_{R} \cap X \neq \emptyset\right\}
\end{aligned}
$$

where $[x]_{R}$ denotes the equivalence class containing $x$ in $R$. The boundary of a set $X$ in $A$ is defined as

$$
\operatorname{Bnd}(X)=\overline{A p r}(X)-\underline{A p r}(X) .
$$

Let $U$ be an approximation space and $X, Y \subseteq U$, then we have:

1. $\underline{A p p}(X) \subseteq X \subseteq \overline{A p p}(X)$,

2. $\overline{\operatorname{App}}(U)=\underline{A p p}(U)=U$,

3. $\overline{\operatorname{App}}(\phi)=\underline{A p p}(\phi)=\phi$,

4. $\overline{A p p}(X \cup Y)=\overline{A p p}(X) \cup \overline{A p p}(Y)$,

5. $\underline{A p p}(X \cap Y)=\underline{A p p}(X) \cap \underline{A p p}(Y)$,

6. $\overline{A p p}(X \cap Y) \subset \overline{A p p}(X) \cap \overline{A p p}(Y)$,

7. $\underline{A p p}(X) \cup \underline{A p p}(Y) \subseteq \underline{A p p}(X \cup Y)$,

8. $\overline{A p p}(X) \subset \overline{A p p}(Y)$ and $\underline{A p p}(X) \subset \underline{A p p}(Y)$ whenever $X \subset Y$.

Example 3. In the following graph, the grid represents an approximation space $A=(U, R)$ partitioned into squares, that is a classification $U / R$ of $U$ is the set of gray squares. Let the red ellipse be a subset $X$ of $U$. So the lower approximation of $X$ is the set of all squares that lay inside the ellipse which colored in green, whereas the upper approximation of $X$ is set of all squares that surrounded by blue lines; because they intersect with ellipse $X$. The boundary of $X$ is the set of all squares that surrounded by blue lines and not green.

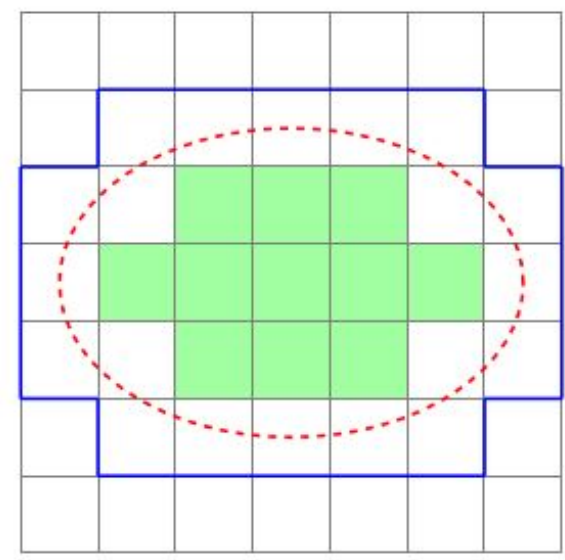

Figure 1: Example of approximation of sets 
In 1994, Biwas constructed the concepts of rough groups and rough subgroups.

Definition 4. A subset $G$ of an approximation space $(U, R)$ with a binary operation $(*)$ defined on $U$ is called a rough group, whenever the following four axioms hold:

1. $x * y \in \overline{\operatorname{App}}(G), \forall x, y \in G$;

2. Associative property holds in $\overline{\operatorname{App}}(G)$;

3. $\exists e \in \overline{A p p}(G)$ such that $\forall x \in G, \quad x * e=e * x=x$, where $e$ is called the rough identity; and

4. $\forall x \in G, \exists y \in G$ such that $x * y=y * x=e$, where $y$ is called the rough inverse, and denoted by $x^{-1}$.

Example 5. Let $X=\mathbb{Z}_{3} \times \mathbb{Z}_{3}=\left\{\langle a, b\rangle: a, b \in \mathbb{Z}_{3}\right\}$ with operation of addition modulo 3 , and let

$$
V=\{\langle 1,1\rangle,\langle 1,2\rangle,\langle 2,2\rangle,\langle 2,1\rangle,\langle 1,0\rangle,\langle 2,0\rangle\} \subseteq X .
$$

The classification of $X$ is $X / R=\left\{C_{1}, C_{2}\right\}$ where $C_{1}$ is the set of all ordered pairs whose at least one component is zero, and $C_{2}=X \backslash C_{1}$. Then $\overline{A p p}(V)=X$.

Let us verify that $V$ is a rough group. From Definition 4, we have the conditions

1. $x * y \in \overline{A p p}(V), \forall x, y \in V$; because $\overline{\operatorname{App}}(V)=X$,

2. associative property already holds in $\overline{A p p}(V)$,

3. $\langle 0,0\rangle \in \overline{A p p}(V)$ is considered as the rough identity,

4. $\langle 1,1\rangle^{-1}=\langle 2,2\rangle \in V,\langle 1,2\rangle^{-1}=\langle 2,1\rangle \in V$, and $\langle 1,0\rangle^{-1}=\langle 2,0\rangle \in V$.

are satisfied, and hence $V$ is a rough group.

Example 6. Let $X=\mathbb{R}$ be the set of real numbers with the usual addition and the classification of $X$ is $X / R=$ $\left\{E_{1}, E_{2}\right\}$ where $E_{1}$ is the set of nonnegative real numbers and $E_{2}=\mathbb{R} \backslash E_{1}$. Let $V=\mathbb{R}-\{0\}$, then $\overline{A p p}(V)=\mathbb{R}$. Now, let us show that $V$ is a rough group. We have to prove that the four conditions in Definition 4 hold.

1. It is clearly that the first condition holds for addition on $V$,

2. the associative property holds in $\overline{A p p}(V)$,

3. 0 is considered as the rough identity,

4. for each $x \in V$, there is $x^{-1}=-x \in V$ such that $x+x^{-1}=0$.

Therefore, $V$ is a rough group.

Example 7. Let $U=\mathbb{Z}_{6}$ be the residue additive group of module 6 , and the binary operation (*) be the addition modulo 6. The classification of $U$ is $U / R=\left\{C_{1}, C_{2}, C_{3}\right\}$ where $C_{1}=\{\overline{0}, \overline{1}, \overline{4}\}, C_{2}=\{\overline{2}, \overline{3}\}$, and $C_{3}=\{\overline{5}\}$. Let $V=\{\overline{1}, \overline{2}, \overline{4}, \overline{5}\} \subset U$. Notice that $\overline{A p p}(V)=U$. We will prove that $V$ is a rough group by Definition 4.

1. Since $\overline{A p p}(V)=U$, then the first condition holds,

2. $(*)$ is associative,

3. $\overline{0} \in \overline{A p p}(V)$ represents the rough identity,

4. $\overline{1}^{-1}=\overline{5} \in V$, and $\overline{2}^{-1}=\overline{4} \in V$.

Hence, $V$ is a rough group

Definition 8. If $G$ is a rough group and $H \subseteq G$, then $H$ is said to be a rough subgroup of $G$ if it is itself a rough group induced with operation of $G$. 
Remark 9. The only trivial rough subgroup of a rough group $G$ is $G$ itself, and the subset $\{e\}$ will be a trivial rough subgroup only if $e \in G$.

Definition 10. A subset $H$ of a rough group $G$ is a rough subgroup of $G$ if and only if

1. $x y \in \overline{A p p}(H) ; \quad \forall x, y \in H$,

2. $x^{-1} \in H ; \quad \forall x \in H$.

In 2016, Nurettin Bağırmaz [1] completed Biswas's work by combine the concepts of topological groups and rough groups, which defined the topological rough groups.

Definition 11. A topological rough group is a rough group $(G, *)$ in an approximation space $A=(U, R)$ endowed with a topology $\tau$ on $\bar{G}$, satisfying two axioms:

1. The map $(x, y) \rightarrow x * y$ from $G \times G$ into $\bar{G}$ is continuous; and

2. The inverse map $x \rightarrow x^{-1}$ from $G \rightarrow G$ is continuous.

Example 12. According to Example 5, let $\mathcal{D}$ be the discrete topology on $\overline{A p r}(V)=X$ and $\mathcal{D}_{V}$ be the induced topology on $V$, so $\mathcal{D}_{V}$ is also a discrete topology on $V$. Let us show that a rough group $V$ with $\mathcal{D}_{V}$ is a topological rough group.

1. First, we claim that the mapping $f: V \times V \rightarrow \overline{A p r}(V)$ is continuous. Let $\{x\} \subset \overline{A p r}(V)=X$ be an arbitrary open set in $X$, then $f^{-1}(\{x\})=\left\{f^{-1}(x)\right\} \in V \times V$ is also an open set in $V \times V$. Thus, $f$ is continuous.

2. Secondly, we need to check that the mapping $g: V \rightarrow V$ which is defined by $g(x)=x^{-1}$ is continuous on $V$. Let $\left\{x^{-1}\right\} \subset V$ be an arbitrary open set in $V$. Then $g^{-1}\left(\left\{x^{-1}\right\}\right)=\left\{g^{-1}\left(x^{-1}\right)\right\}=\{x\}$ is also an open set in $V$, and hence $g$ is continuous on $V$.

Therefore the rough group $V$ induced with $\mathcal{D}_{V}$ is a topological rough group by Definition 11.

Example 13. In Example 6 we have seen that $V=\mathbb{R}-\{0\}$ is a rough group. Let $\mathcal{U}$ be the usual topology on $\mathbb{R}$ and $\mathcal{U}_{V}$ be the induced topology on $V$. Let us verify that $V$ endowed with $\mathcal{U}_{V}$ represents a topological rough group. The base of the topology $\mathcal{U}_{V}$ on $V$ is

$$
\mathcal{B}_{V}=\{(a, b) \cap V: a, b \in \mathbb{R} ; a<b\},
$$

where it is again a usual topology on $V$. So, the basic open set for the product topology on $V \times V$ takes the form

$$
((a, b) \times(c, d)) \cap V^{2} ;(a, b),(c, d) \in \mathcal{U},
$$

which represents an open rectangle on $\mathbb{R}^{2}$ except $x$ and $y$-axes. So, the topology on $V \times V$ is also the product topology on $V \times V$.

1. We want to check that the mapping $f: V \times V \rightarrow \overline{A p r}(V)=\mathbb{R} ; \quad f(\langle x, y\rangle)=x+y$ is continuous on $V \times V$. Let $(a, b)$ be an arbitrary basic open set in $(\mathbb{R}, \mathcal{U})$. Then $f^{-1}((a, b))=\{\langle x, y\rangle: a<x+y<b\}$ and it is not an open set in $V \times V$, for example the open interval $(-1,1)$ is a basic open set in $\mathbb{R}$ but its inverse image is the set $\left\{\langle x, y\rangle \in \mathbb{R}^{2}:-1<x+y<1\right\}$ and it is not subset of $V \times V$ since it contains the element $\langle 0,0\rangle$.

Hence, $V$ endowed with the induced usual topology on $\mathbb{R}$ does not represent a topological rough group.

Example 14. Example 7 shown that $V=\{0,1,2,3,4\}$ is a rough group. In this example we want to show that $V$ together with the indiscrete topology is a topological rough group. Let $\mathcal{I}$ be the indiscrete topology on $\overline{A p r}(V)=\mathbb{Z}_{6}$, then the induced topology $\mathcal{I}_{V}$ on $V$ and the product topology $\mathcal{I}_{V \times V}$ on $V \times V$ are also the indiscrete topologies on $V$ and $V \times V$, respectively. Now, we shall verify the conditions in Definition 11

1. The map $f: V \times V \rightarrow \overline{A p r}(V)$ is continuous since the inverse image of each open set in $\mathcal{I}$ is open in $\mathcal{I}_{V \times V}$,

2. also the map $f: V \rightarrow V$ that defined by $f(x)=x^{-1}$ is continuous for the same reason.

Therefore, $V$ with the indiscrete topology $\mathcal{I}_{V}$ represents a topological rough group. 


\section{Rough Vector Spaces}

In this section, the definition of a rough vector space and its related properties and examples will be given.

Definition 15. Let $(U, R)$ be an approximation space. A subset $V \subseteq U$ is called a rough vector space over a field $\mathbb{F}$, denoted by $\boldsymbol{R} \boldsymbol{V}_{\mathbb{F}}$, if $V$ is a rough group under the binary operation $(+)$ and the commutative property of $(+)$ holds in $\overline{A p p}(V)$. Moreover, for all $\lambda \in \mathbb{F}$ and $v \in V$, there is an element $\lambda \cdot v \in \overline{A p p}(V)$ such that the following axioms are satisfied for all $\alpha, \beta \in \mathbb{F}$ and all $u, v \in V$ :

1. $\alpha \cdot(u+v)=\alpha \cdot u+\alpha \cdot v$;

2. $(\alpha+\beta) v=\alpha \cdot v+\beta \cdot v$;

3. $\alpha \cdot(\beta \cdot v)=(\alpha \cdot \beta) \cdot v$; and

4. $1 \cdot v=v$, where $1 \in \mathbb{F}$.

Definition 16. Let $A=(U, R)$ be an approximation space and let $V \subseteq U$ be a rough vector space over the field $\mathbb{F}$. $A$ nonempty subset $X \subset V$ is called its rough subspace over the same field $\mathbb{F}$ whenever it is a rough vector space itself with respect to the same operations those defined on $V$.

Definition 17. A subset $W$ of a rough vector space $V$ over the field $\mathbb{F}$ is a rough subspace if and only if these two conditions below are satisfied,

1. $W$ is a rough subgroup,

2. $\lambda x \in \overline{A p p}(W) ; \forall x \in W$ and $\forall \lambda \in \mathbb{F}$.

Example 18. Let $X=\mathbb{Z}_{3} \times \mathbb{Z}_{3}=\left\{(a, b): a, b \in \mathbb{Z}_{3}\right\}$ with operation of addition modulo 3 , and let

$$
V=\{(1,1),(1,2),(2,2),(2,1),(1,0),(2,0)\} \subseteq X .
$$

The classification of $U$ is as in Example 5. Let us verify that $V$ is a rough vector space over the field $\mathbb{Z}_{3}$ with multiplication modulo 3. We have already showed that $V$ is a rough group, and it is strightforward to check that the addition $(\bmod 3)$ is commutative on $\overline{A p p}(V)=X$. Also, for each $\lambda \in \mathbb{Z}_{3}$, we have $\lambda v \in \overline{A p p}(V)$. So it just remains to show that the four conditions of scalar multiplication in Definition 15 are true. Let $\alpha, \beta \in \mathbb{Z}_{3}$ and $u, v \in V$ be arbitrary. Then

1. The multiplication $(\bmod 3)$ is distributive over addition $(\bmod 3)$, $\llbracket \alpha \rrbracket_{3}\left(\llbracket u \rrbracket_{3}+_{3} \llbracket v \rrbracket_{3}\right)=\llbracket \alpha \rrbracket_{3}\left(\llbracket u+v \rrbracket_{3}\right)=\llbracket \alpha(u+v) \rrbracket_{3}=\llbracket \alpha u+\alpha v \rrbracket_{3}=\llbracket \alpha u \rrbracket_{3}+{ }_{3} \llbracket \alpha v \rrbracket_{3}=\llbracket \alpha \rrbracket_{3}\left(\llbracket u \rrbracket_{3}\right)+{ }_{3}$ $\llbracket \alpha \rrbracket_{3}\left(\llbracket v \rrbracket_{3}\right)$,

2. $\left(\llbracket \alpha \rrbracket_{3}+{ }_{3} \llbracket \beta \rrbracket_{3}\right) \llbracket u \rrbracket_{3}=\left(\llbracket \alpha+\beta \rrbracket_{3}\right) \llbracket u \rrbracket_{3}=\llbracket\left(\alpha+\beta u \rrbracket_{3}=\llbracket \alpha u+\beta\right) u \rrbracket_{3}=\llbracket \alpha u \rrbracket_{3}+{ }_{3} \llbracket \beta u \rrbracket_{3}$,

3. $\llbracket \alpha \rrbracket_{3}\left(\llbracket \beta \rrbracket_{3} \llbracket v \rrbracket_{3}\right)=\llbracket \alpha \rrbracket_{3}\left(\llbracket \beta v \rrbracket_{3}\right)=\llbracket \alpha(\beta v) \rrbracket_{3}=\llbracket(\alpha \beta) v \rrbracket_{3}=\llbracket\left(\alpha \beta \rrbracket_{3} \llbracket v \rrbracket_{3}=\left(\llbracket \alpha \rrbracket_{3} \llbracket \beta \rrbracket_{3}\right) \llbracket v \rrbracket_{3}\right.$,

4. there is $1 \in \mathbb{Z}_{3}$ such that for each $v \in V, 1 v=v$.

Thus, $V$ is a rough vector space over $\mathbb{Z}_{3}$.

Example 19. Let $A$ be the approximation space in Example 6, and $V$ is the rough group in the same example. We want to prove that $V$ with the usual addition and multiplication is also a rough vector space over the field $\mathbb{R}$. So, we have to check the commutative property for addition on $\overline{A p p}(V)=\mathbb{R}$ and the conditions of the field in Definition 15 . Of course $(+)$ is commutative and the conditions

1. $\lambda x \in \overline{A p p}(V), \forall \lambda, x \in \mathbb{R}$,

2. $\alpha(x+y)=\alpha x+\alpha y ; \forall x, y \in V, \forall \alpha \in \mathbb{R}$,

3. $(\alpha+\beta) x=\alpha x+\beta x ; \forall \alpha, \beta \in \mathbb{R}$, and $\forall x \in V$,

4. $\alpha(\beta x)=(\alpha \beta) x ; \forall \alpha, \beta \in \mathbb{R}$, and $\forall x \in V$,

5. $1 \in \mathbb{R}$ is a multiplicative identity,

hold, and hence $V$ is a rough vector space. 
Example 20. Let $U=\mathbb{Z}_{6}$ be the residue additive group of module 6 . The classification of $U$ is $U / R=\left\{C_{1}, C_{2}, C_{3}\right\}$ where $C_{1}=\{\overline{0}, \overline{1}, \overline{4}\}, C_{2}=\{\overline{2}, \overline{3}\}$, and $C_{3}=\{\overline{5}\}$. Let $V=\{\overline{1}, \overline{2}, \overline{4}, \overline{5}\} \subset U$. In Example 7, we have seen $V$ is a rough group under the addition of residue $(\bmod 6)$. Let us show that $V$ is also a rough vector space over the field $\mathbb{Z}_{3}$ with the multiplication of residue $(\bmod 3)$. Notice that $\overline{A p p}(V)=U$, so for all $\lambda \in \mathbb{Z}_{3}$ and $v \in V$ we have $\lambda x \in \overline{A p p}(V)$. It is straightforward to check that the conditions 1,2,3 and 4 in definition 15 hold. Therefore, $V$ is a rough vector space.

Theorem 21. If $V$ is a rough vector space and $V=\overline{A p p}(V)$, then $V$ is also a vector space.

Proof. The proof is straightforward. According to Definition 15, $\mathrm{V}$ satisfy the conditions of vector spaces.

Remark 22. The intersection of two rough vector spaces does not have to be a rough vector subspace. For example, let $U=\mathbb{Z}_{6} \times \mathbb{Z}_{6}$ and the classification of $U$ is $U / R=\left\{C_{1}, C_{2}\right\}$ where $C_{1}$ consists all element that has at least one component is zero and $C_{2}=U \backslash C_{1}$. Let $V=\{(1,1),(0,2),(0,4),(5,5)\}$ and $W=\{(1,1),(5,5),(3,3),(4,0),(2,0)\}$. Then $V$ and $W$ are both rough vector spaces, whereas their intersection $V \cap W=\{(1,1),(5,5)\}$ with approxiamtion $\overline{A p p}(V \cap W)=C_{2}$ is not a rough vector space since $\overline{A p p}(V \cap W)=C_{2}$ has no any rough identity.

In the next proposition we provide the condition how to be the intersection of two rough vector spaces is a rough vector space.

Proposition 23. If $(U, R)$ is an approximation space and $V$ and $W$ are two rough vector spaces over the field $\mathbb{F}$ with the binary operation $(+)$ and scalar multiplication $(\cdot)$, then $V \cap W$ is also a rough vector subspace over the same field whenever

$$
[\overline{A p p}(V) \cap \overline{A p p}(W)] \subset \overline{A p p}(V \cap W)
$$

Proof. To show that $V \cap W$ is a rough vector subspace, we have to prove that $V \cap W$ is a rough subgroup and $\lambda x \in \overline{A p p}(V \cap W)$ for all $\lambda \in \mathbb{F}$ and $x \in V \cap W$. Let $x$ and $y$ be arbitrary elements in $V \cap W$ and $\lambda \in \mathbb{F}$ be arbitrary. So, $x+y$ is in both $\overline{A p p}(V)$ and $\overline{A p p}(W)$ because $V$ and $W$ are rough vector spaces. Then $x+y \in \overline{A p p}(V) \cap \overline{A p p}(W)$ and by 3.1 we have $x+y \in \overline{A p p}(V \cap W)$. Also $x^{-1} \in V \cap W$, and hence by Definition $10 V \cap W$ is a rough subgroup. Now, if $x \in V \cap W$ and $\lambda \in \mathbb{F}$, then $\lambda x$ is in both $\overline{A p p}(V)$ and $\overline{A p p}(W)$. So, $\lambda x \in \overline{A p p}(V) \cap \overline{A p p}(W)$ and again by our assumption 3.1 we get $\lambda x \in \overline{A p p}(V \cap W)$. Therefore, by Definition $17 V \cap W$ is a rough vector subspace.

\section{Algebraic Structure}

Definition 24. Let $A=(U, R)$ be an approximation space. A rough vector space $V$ is called the direct sum of its rough vector subspaces $W_{1}$ and $W_{2}$ if

1. $\overline{A p p}\left(W_{1}\right) \cap \overline{A p p}\left(W_{2}\right)=\{0\}$, and

2. every element $x$ in $V$ can be written as $x=w_{1}+w_{2}$ where $w_{1} \in \overline{A p p}\left(W_{1}\right)$ and $w_{2} \in \overline{A p p}\left(W_{2}\right)$.

Note that in the first condition, 0 is the rough identity of the rough group $(V,+)$.

Definition 25. Let $A=(U, R)$ be an approximation space and let $V$ and $W$ be two rough vector spaces on $A$ over the same field $\mathbb{F}$. A mapping $L: \overline{A p p}(V) \rightarrow \overline{A p p}(W)$ is said to be rough linear if the followings hold:

1. $L(u+v)=L(u) * L(v) ;$ and

2. $L(\alpha v)=\alpha L(v)$

where + is the binary operation that defined on $V$ and $*$ is the binary operation that defined on $W$.

Definition 26. A rough linear map is called a rough isomorphism if it is bijective.

Definition 27. Let $A=(U, R)$ be an approximation space and let $L: \overline{A p p}(V) \rightarrow \overline{A p p}(W)$ be a rough linear map. Then the set

$$
\operatorname{RKer}(L)=\{v \in \overline{A p p}(V): L(v)=0 \overline{\overline{A p p}(W)}\}=L^{-1}(0 \overline{\overline{A p p}(W)})
$$

is called the rough kernel of $L$, where $0_{\overline{A p r}(W)}$ is the rough identity of $W$. 
Remark 28. If $L: \overline{A p p}(V) \rightarrow \overline{A p p}(W)$ is a rough isomorphism, then $L^{-1}: \overline{A p p}(W) \rightarrow \overline{A p p}(V)$ is also. Since the linearity property holds for inverse of rough isomorphism $L^{-1}$.

Definition 29. Let $V$ be a rough vector space over the field $\mathbb{F}$ in an approximation space $(U, R)$. Let $N$ be a rough subspace of $V$. The rough quotient of $V$ by $N$ is the set

$$
V / N=\{x+\overline{A p p}(N): x \in \overline{A p p}(V)\}
$$

where $x+N$ is the set of all $y$ that satisfying the relation $x-y \in \overline{A p p}(N)$.

Definition 30. Let $V$ be a rough vector space in an approximation space $(U, R)$ over the field $\mathbb{F}$, with binary operation $(+)$. Let $N$ be a rough subspace of $V$. Then the set

$$
x+\overline{A p p}(N)=\{x+n: n \in \overline{A p p}(N)\}
$$

is called the rough coset of $N$ in $V$ containing $x$, where $x$ is a fixed element in $\overline{A p p}(V)$.

Note that, by commutative property of $\overline{A p p}(V)$, we have that

$$
x+\overline{A p p}(N)=\overline{A p p}(N) ; \quad \forall x \in \overline{A p p}(V),
$$

and there is no difference between these two expressions.

Theorem 31. If $V / N$ is the rough quotient of $V$ by $N$. Then

$$
\overline{A p p}(V)=\bigcup V / N
$$

Proof. It is trivial to say that $\bigcup V / N \subseteq \overline{A p p}(V)$. We just have to prove that $\overline{A p p}(V) \subseteq \bigcup V / N$. Let $x \in \overline{A p p}(V)$ be an arbitrary. Then for each $N \subseteq V, \exists 0 \in \overline{A p p}(N)$ such that $x=x+0 \in x+\overline{A p p}(N)$. So, $\overline{A p p}(V) \subseteq \cup V / N$.

Lemma 32 (Properties of Rough Cosets). Let $N$ be a rough subspace of a rough vector space $V$ and let $x, y \in \overline{A p p}(V)$.

1. $x \in x+\overline{A p p}(N)$,

2. $x+\overline{A p p}(N)=\overline{A p p}(N)$ if and only if $x \in \overline{A p p}(N)$,

3. $x+\overline{A p p}(N)=y+\overline{A p p}(N)$ if and only if $x \in y+\overline{A p p}(N)$,

4. $x+\overline{A p p}(N)=y+\overline{A p p}(N)$ or $(x+\overline{A p p}(N)) \cap(y+\overline{A p p}(N))=\emptyset$,

5. $x+\overline{A p p}(N)=y+\overline{A p p}(N)$ if and only if $x-y \in \overline{A p p}(N)$,

6. $|x+\overline{A p p}(N)|=|y+\overline{A p p}(N)|$, whenever $V$ is finite,

7. $x+\overline{A p p}(N)$ is a rough subspace of $V$ if and only if $x \in \overline{A p p}(N)$.

Proof. $\quad 1$. Since $0 \in \overline{A p p}(N)$, then $x=x+0 \in x+\overline{A p p}(N)$.

2. Assume $x+\overline{A p p}(N)=\overline{A p p}(N)$, by the previous property $x \in x+\overline{A p p}(N)=\overline{A p p}(N)$. Conversely, assume that $x \in \overline{A p p}(N)$. Since $\overline{A p p}(N)$ is a rough vector space, then $x+\overline{A p p}(N)=\overline{A p p}(N)$.

3. Assume $x+\overline{A p p}(N)=y+\overline{A p p}(N)$. We claim $x \in y+\overline{A p p}(N)$. By the first property, $x \in x+\overline{A p p}(N)=y+$ $\overline{A p p}(N)$. Conversely, assume $x \in y+\overline{A p p}(N)$ and we claim $x+\overline{A p p}(N)=y+\overline{A p p}(N)$. Then by our assumption $x=y+n$, where $n \in \overline{A p p}(N)$, and so $x+\overline{A p p}(N)=(y+n)+\overline{A p p}(N)=y+(n+\overline{A p p}(N))=y+\overline{A p p}(N)$. Thus the equality holds.

4. Suppose that there is an element $z \in(x+\overline{A p p}(N)) \cap(y+\overline{A p p}(N))$. Then $z \in x+\overline{A p p}(N)$ and $z \in y+\overline{A p p}(N)$. So, by the third property we get $z+\overline{A p p}(N)=x+\overline{A p p}(N)=y+\overline{A p p}(N)$. Thus, any two cosets are equal or disjoint.

5. $x+\overline{A p p}(N)=y+\overline{A p p}(N)$ if and only if $(x-y)+\overline{A p p}(N)=0+\overline{A p p}(N)$ if and only if $(x-y) \in 0+\overline{A p p}(N)=$ $\overline{A p p}(N)$. 
6. To show this property, we must find a bijection from $x+\overline{A p p}(N)$ onto $y+\overline{A p p}(N)$. Obviously, the function

$$
x+n \rightarrow y+n ; \quad n \in \overline{A p p}(N)
$$

that maps $x+\overline{A p p}(N)$ onto $y+\overline{A p p}(N)$, and if $y+n_{1}=y+n_{2}$, then $n_{1}=n_{2}$ and it is one-to-one. Hence, $|x+\overline{A p p}(N)|=|y+\overline{A p p}(N)|$.

7. Assume $x+\overline{A p p}(N)$ is a rough subspace of $V$. Since $x=x+0 \in x+\overline{A p p}(N), 0 \in \overline{A p p}(N)$, and $\overline{A p p}(N)$ is a rough subspace, then by Definition 15 we have $x=x+0 \in \overline{A p p}(N)$. Conversely, if $x \in \overline{A p p}(N)$, so by the second property we get $x+\overline{A p p}(N)=\overline{A p p}(N)$ and $\overline{A p p}(N)$ is already a rough subspace and so $x+\overline{A p p}(N)$ is also.

Definition 33. Let $V$ be a rough vector space in an approximation space $(U, R)$. A subset $F \subset \overline{A p p}(V)$ is called symmetric if for each $x \in F,(-x) \in F$.

Corollary 34. If $V$ is a rough vector space in an approximation sapce $(U, R)$, then $V$ and every rough subspace of $V$ is a symmetric set.

\section{Topological Rough Vector Spaces}

Definition 35. Let $V$ be a rough vector space over the field $\mathbb{F}$. Then a topological rough vector space, denoted by $\boldsymbol{T R} \boldsymbol{V}_{\mathbb{F}}$, is $V$ together with an induced topology $\tau_{V}$ that introduced by a topology on $\overline{A p r}(V)$, satisfying two axioms:

1. The map $+: V \times V \rightarrow \overline{A p r}(V),(x, y) \rightarrow x+y$ is continuous, and

2. the map $: \mathbb{F} \times V \rightarrow \overline{A p r}(V)$ is also continuous.

Then it is written as the pair $\left(V, \tau_{V}\right)$. Here the topology defined on $\mathbb{F}$ is the standard topology.

Example 36. In Example 18 we have seen that $V=\{(1,1),(1,2),(2,2),(2,1),(1,0),(2,0)\}$ is a rough vector space in the approximation space $(X, R)$. We want to verify that $V$ together with the discrete topology $\mathcal{D}_{V}$ is a topological rough verctor space by checking conditions in Definition 35. Obviously, the first condition was satisfied in Example 12. So, it remains to check the continuity of the function $\cdot: \mathbb{Z}_{3} \times V \rightarrow \overline{A p r}(V)$. The topology that defined on the field $\mathbb{Z}_{3}$ is the standard topology on the finite set which is the discrete topology, so the product topology on $\mathbb{Z}_{3} \times V$ is the discrete topology too. Since every function from a discrete space is continuous the condition holds, and therefore the rough vector space $V$ over the field $\mathbb{Z}_{3}$ together with a discrete topology represents a topological rough vector space.

Example 37. According to Example 20, $V=\{1,2,4,5\}$ over the field $\mathbb{Z}_{3}$ is a rough vector space and in Example 14 we have seen that $V$ with indiscrete topology represents a topological rough group. To show that a rough vector space $V$ over $\mathbb{Z}_{3}$ with the indiscrete topology is a topological rough vector space we have to prove that the function $f: \mathbb{Z}_{3} \times V \rightarrow \overline{A p r}(V)$ is continuous. The product topology on $\mathbb{Z}_{3} \times V$ is $\left\{\emptyset, \mathbb{Z}_{3} \times V\right\} \cup\left\{U \times V: U \subset \mathbb{Z}_{3}\right\}$, where the topology on $\mathbb{Z}_{3}$ is the discrete topology. Now, let $U$ be an arbitrary open set in $\overline{A p r}(V)$, since the topology that defined on $\overline{A p r}(V)$ is the indiscrete topology so we have two cases,

Case(1): if $U=V$, then $f^{-1}=F \times V$ such that $F \subseteq \mathbb{Z}_{3}$, and of course it is open in $\mathbb{Z}_{3} \times V$.

Case(2): if $U=\emptyset$, then $f^{-1}(U)=\emptyset$ where it is open in $\mathbb{Z}_{3} \times V$.

Therefore, $f: \mathbb{Z}_{3} \times V \rightarrow \overline{A p r}(V)$ is continuous and hence our claim has proved.

Proposition 38. Let $\left(V, \tau_{V}\right)$ be a topological rough vector space over the field $\mathbb{F}$. For a fixed point a $\in \mathbb{R}$ and a fixed scalar $\alpha \in \mathbb{F}$ let $T_{a}: \mathbb{R} \rightarrow \mathbb{R}$ and $M_{\alpha}: V \rightarrow V$ be the translation and the multiplication operator which are defined by

$$
\begin{gathered}
T_{a}(x)=x+a \\
M_{\alpha}(x)=\alpha \cdot x,
\end{gathered}
$$

respectively. Then these functions are both homeomorphisms of $V$ onto $V$.

Proof. The functions $T_{a}$ and $M_{\alpha}$ are One-to-One by the definition 15 and they are onto since for each $x \in V$, there are $0 \in V$ and $1 \in \mathbb{F}$ such that $x+0=x$ and $1 \cdot x=x$. So, $T_{a}$ and $M_{\alpha}$ are bijections. Moreover, by the Definition 35 they are continuous, and their inverses $T_{-a}, M_{1 / \alpha}$ are so because $-a \in V$ and $\frac{1}{\alpha} \in \mathbb{F}$. Hence, $T_{a}$ and $M_{\alpha}$ are both homeomorphisms. 
Theorem 39. Let $\left(V, \tau_{V}\right)$ and $\left(W, \tau_{W}\right)$ be topological rough vector spaces and the rough linear map $f: \overline{A p r}(V) \rightarrow$ $\overline{A p r}(W)$ is continuous whereas $\overline{A p r}(V)$ is a first-countable space. Then the rough kernel Rker $(f)$ is closed.

Proof. The desired argument is that the rough kernel contains all its limit points. Assume that $f$ is continuous on $\overline{A p r}(V)$ and it is rough linear. Let $\left(x_{n}\right)$ be the sequence in $\operatorname{Rker}(f)$ that converges to $x \in \overline{A p r}(V)$. By continuity of $f, f\left(x_{n}\right)$ converges to $f(x)$. Since $x_{n} \in R k e r(f)$,

$$
f(x)=\lim _{n \rightarrow \infty} f\left(x_{n}\right)=0 .
$$

Then $x \in \operatorname{Rker}(f)$ and hence $\operatorname{Rker}(f)$ is closed.

\section{References}

[1] N. Bağırmaz, İçen, İ., A.F. Özcan, Topological Rough Groups. Topological Algebra and its Applications, 4(1) 2016, 31-38. https://doi.org/10.1515/taa-2016-0004

[2] R. Biwa, S. Nanda, Rough Groups and Rough Subgroups, Bulletin of the Polish Academy of SciencesMathematics, 42(3)1994, 251-254.

[3] B. Davvaz, Roughness in rings, Information Sciences, 164(1-4) 2004, 147-163. Https://Doi.Org/10.1016/J.Ins.2003.10.001

[4] B. Davvaz, M. Mahdavipour, Roughness in modules, Information Sciences, 176(24) 2006, 3658-3674. https://doi.org/10.1016/j.ins.2006.02.014

[5] Z. Pawlak, Rough sets, International journal of computer \& information sciences, 11(5) 1982, 341-356. 